EPJ Web of Conferences 60, 20016 (2013)

DOI: $10.1051 /$ epjconf $/ 20136020016$

(C) Owned by the authors, published by EDP Sciences, 2013

\title{
Search for stable mutli-charged particles with the ATLAS experiment
}

\author{
Simone Zimmermann on behalf of the ATLAS Collaboration ${ }^{1, a}$ \\ ${ }^{1}$ Physikalisches Institut, University of Bonn, Nussallee 12, 53115 Bonn, Germany
}

\begin{abstract}
A search for long-lived, multi-charged particles is performed using the ATLAS detector. We examine $p p$ collision-data taken during the 2011 LHC running, corresponding to an integrated luminosity of $4.4 \mathrm{fb}^{-1}$. A search is made for charged particle tracks exhibiting anomalously high ionization consistent with stable massive particles with charges from $|q|=2 e$ to $|q|=6 e$. For this search new variables of specific energy loss per path length $(\mathrm{d} E / \mathrm{d} x)$ are developed and used in the candidate selection. No excess is observed with respect to the prediction of Standard Model processes. The 95\% CL cross-section limits are interpreted as lower mass limits of 420-490 GeV for a simplified Drell-Yan production model.
\end{abstract}

\section{Introduction}

A search for stable massive particles carrying electric charges in the range of $|q|=2 e$ to $|q|=6 e[1]$ is performed with the ATLAS detector [2] at the LHC [3]. Motivation for this analysis arises from an unexploited search regime for particles with charges $|q|=2 e$ to $|q|=5 e$ [4]. The high masses and hence low values of $\beta \gamma$ as well as the multiple charges imply high energy losses through interactions with the detector material. Consequently, the analysis follows a signature-based approach seeking out charged particle tracks showing anomalously high ionization losses in three ATLAS subdetectors of the inner tracking detectors and the muon system. An observation of such particles would be a clear indication for physics beyond the Standard Model. Theoretical motivation is given by models of almost commutative geometry [5] and minimal walking technicolor [6] predicting stable multi-charged particles. For the Monte Carlo production, a simplified DrellYan model without $Z$ boson exchange is used to generate generic particle pairs of mass 50 to $600 \mathrm{GeV}$ with electric charges $|q|=2 e$ to $|q|=6 e$.

\section{Selection}

The complete 2011 ATLAS dataset of proton-proton collisions recorded at $\sqrt{s}=7 \mathrm{TeV}$ is analyzed. Events are selected by a single muon trigger with $p_{\mathrm{T}}>18 \mathrm{GeV}$, which represents the lowest available $p_{\mathrm{T}}$ threshold in 2011. The resulting data sample corresponds to an integrated luminosity of $4.4 \mathrm{fb}^{-1}$. The analysis selects central muon signatures with a transverse momentum of at least $20 \mathrm{GeV}$ in the inner tracking detector. Furthermore, tracks are selected that are produced close to the primary vertex and have sufficient measurements in the inner tracking detectors.

\footnotetext{
ae-mail: Simone.Zimmermann@ cern.ch
}

\subsection{Energy loss estimators}

In total, three different $\mathrm{d} E / \mathrm{d} x$ measurements from two ATLAS subdetectors in the inner detector, Pixel and TRT, and one in the muon system, MDT, are used in the analysis. The silicon Pixel detector is the innermost ATLAS subdetector and consists of $\sim 80$ million pixels with the capability to measure directly the deposited charge in the detector. $\mathrm{d} E / \mathrm{d} x$ is constructed from this charge measurement. The TRT and MDT are both gas detectors for which a $\mathrm{d} E / \mathrm{d} x$ variable can be constructed from the direct detector readout. The TRT and MDT $\mathrm{d} E / \mathrm{d} x$ variables are developed for this search.

\subsection{Significance variable}

To discriminate against the standard model muon background, a $\mathrm{d} E / \mathrm{d} x$ significance variable

$$
S(\mathrm{~d} E / \mathrm{d} x)=\frac{\mathrm{d} E / \mathrm{d} x_{\text {track }}-\left\langle\mathrm{d} E / \mathrm{d} x_{\mu}\right\rangle}{\sigma\left(\mathrm{d} E / \mathrm{d} x_{\mu}\right)}
$$

is defined, where $\left\langle\mathrm{d} E / \mathrm{d} x_{\mu}\right\rangle$ is the mean $\mathrm{d} E / \mathrm{d} x$ expectation for muons and $\sigma\left(\mathrm{d} E / \mathrm{d} x_{\mu}\right)$ its resolution. $\left\langle\mathrm{d} E / \mathrm{d} x_{\mu}\right\rangle$ and $\sigma\left(\mathrm{d} E / \mathrm{d} x_{\mu}\right)$ are estimated from $Z \rightarrow \mu \mu$ tuning samples for each $\mathrm{d} E / \mathrm{d} x$ variable individually in data and in simulation.

\subsection{Signal selection}

In the following a distinction has to be made between the search for stable massive particles with charge $|q|=2 e$ and $|q|>2 e$. While the former can be selected very efficiently by a cut on $\mathrm{S}($ Pixel $\mathrm{d} E / \mathrm{d} x$ ) $>10$ (see figure 1a), the Pixel detector cannot be used in the search for $|q|>2 e$ due to saturation effects that lead to the loss of efficiency. Alternatively, the TRT high threshold fraction, $f^{\mathrm{HT}}$, giving the fraction of high charge measurements on a TRT track, serves as an excellent variable for all but the charge 
$|q|=2 e$ particle tracks. Figure $1 \mathrm{~b}$ illustrates the poor separation for $Z \rightarrow \mu \mu$ Monte Carlo samples and the mass $200 \mathrm{GeV}|q|=2 e$ signal point. A cut at $f^{\mathrm{HT}}>0.4$ efficiently selects all signal samples with $|q|>2 e$.

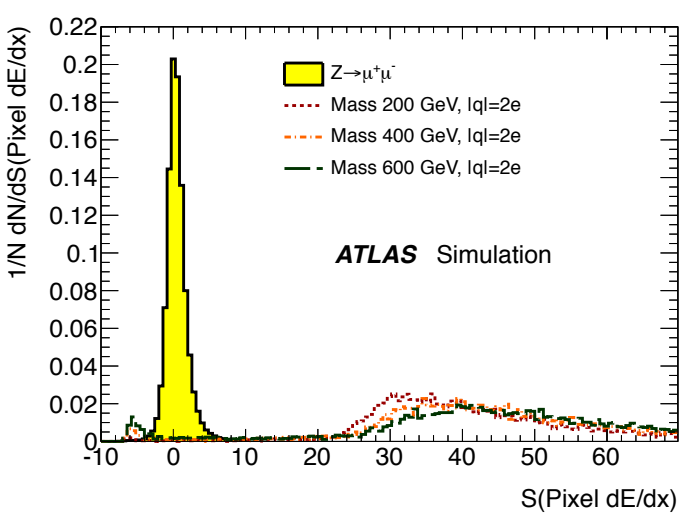

(a) $\mathrm{S}($ Pixel $\mathrm{d} E / \mathrm{d} x)$

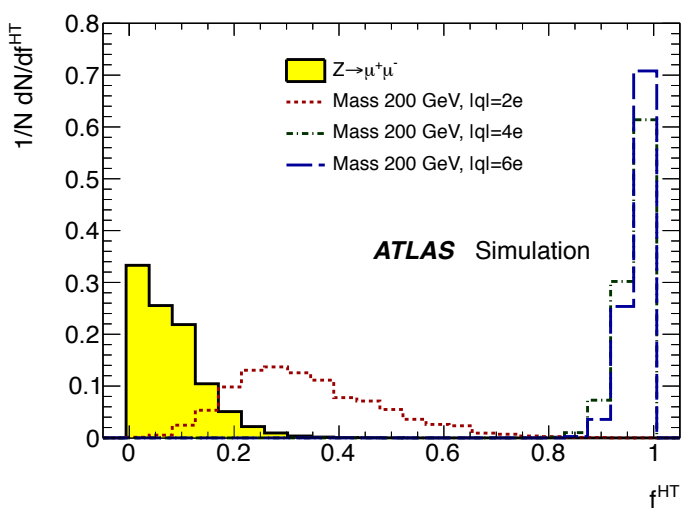

(b) $f^{\mathrm{HT}}$

Figure 1: (a) The simulated distributions of $\mathrm{S}(\operatorname{Pixel} \mathrm{d} E / \mathrm{d} x$ ) for $Z \rightarrow \mu \mu$ and $|q|=2 e$ particles. (b) The TRT high threshold fraction for $Z \rightarrow \mu \mu$ simulation and signal samples of mass $200 \mathrm{GeV}$ and $|q|=2,4,6 e$.

The further selection is based on the TRT and MDT $\mathrm{d} E / \mathrm{d} x$ significances, S(TRT $\mathrm{d} E / \mathrm{d} x)$ and S(MDT $\mathrm{d} E / \mathrm{d} x$ ). Cuts at $\mathrm{S}(\mathrm{TRT} \mathrm{d} E / \mathrm{d} x)>4(5)$ and $\mathrm{S}($ MDT $\mathrm{d} E / \mathrm{d} x)>3(4)$ define the signal region for $|q|=2 e(|q|>2 e)$. Figure 2 illustrates this definition of the signal region $\mathrm{D}$ and the background regions $\mathrm{A}, \mathrm{B}$ and $\mathrm{C}$ for the selected data tracks in the $|q|=2 e$ and $|q|>2 e$ search and simulated signal samples of mass $200 \mathrm{GeV}$ and $|q|=2 e$ and $|q|=4 e$.

\subsection{Background estimation}

The expected number of background candidates in the signal region $\mathrm{D}$ is estimated assuming uncorrelated variables S(TRT $\mathrm{d} E / \mathrm{d} x$ ) and S(MDT $\mathrm{d} E / \mathrm{d} x$ ) via

$$
N^{\mathrm{D}}=\frac{N^{\mathrm{A}} N^{\mathrm{C}}}{N^{\mathrm{A}}},
$$

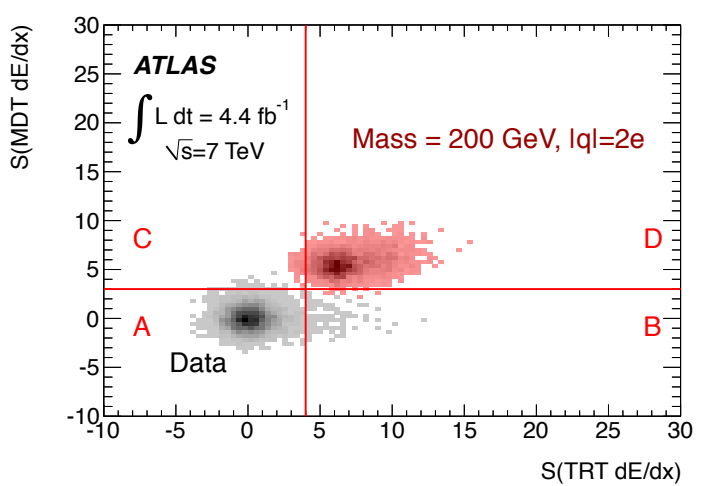

(a) $|q|=2 e$

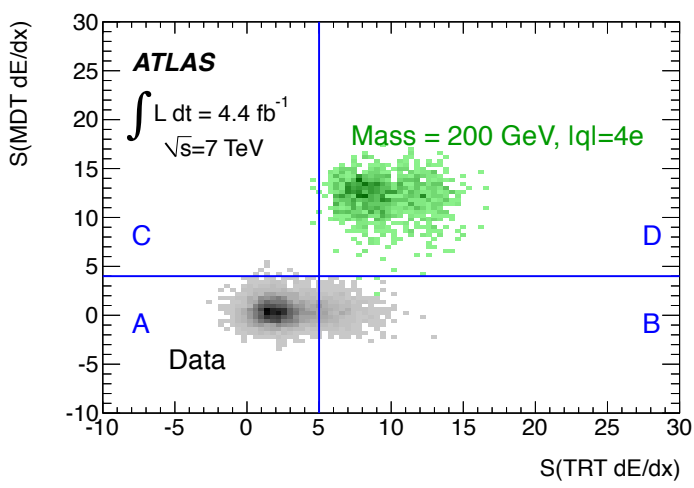

(b) $|q|>2 e$

Figure 2: The plane of S(TRT $\mathrm{d} E / \mathrm{d} x)$ and $\mathrm{S}($ MDT $\mathrm{d} E / \mathrm{d} x$ ) defining the signal region $\mathrm{D}$ for all data recorded in 2011 (black) and a signal of mass $200 \mathrm{GeV}$ and (a) charges $|q|=$ $2 e$ (red), (a) charges $|q|=4 e$ (green).

where $N^{\mathrm{i}}$ is the measured number of background candidates in region $\mathrm{i}$. The resulting background expectations are $0.41 \pm 0.08$ candidates for $|q|=2 e$ and $1.37 \pm 0.46$ candidates for $|q|>2 e$. The uncertainties are statistical.

\subsection{Selection efficiencies}

Signal selection efficiencies are estimated from simulation to be in the range of $0.01 \%$ to $12.6 \%$. These include the detector acceptance, trigger efficiency, and signal selection efficiencies, where the trigger efficiency includes a scaling factor to correct for mismodeled timing distributions of the muon trigger in simulation.

\subsection{Systematic uncertainties}

The dominant systematic uncertainties on the selection efficiencies arise from limited Monte Carlo statistics, the trigger efficiency scaling and the variation of all applied cuts within their uncertainties. The resulting uncertainties on the selection efficiencies amount to $6 \%$ to $25 \%$ depending on the charge. Furthermore, a $5 \%$ uncertainty on the background estimation and $3.8 \%$ on the integrated luminosity are taken into account. 


\section{Experimental challenges}

This analysis faces some unusual experimental challenges due to the high masses and the high charges of the particles. For one, the requirement to reach the muon system within the time of the active detector readout imposes an effective cut on the velocity of the multi-charged particles of $\beta>0.7$. As can be deduced from figure 3 , this leads to high efficiency losses of the signal samples with higher masses as they tend to be produced at lower velocities. Moreover, the cut on $\beta$ translates into a minimal momentum of the particles that can be as high as $200-300 \mathrm{GeV}$ in some cases.

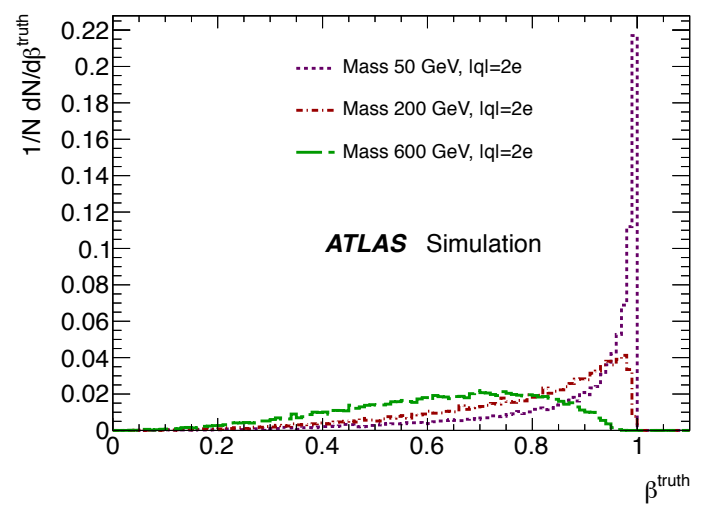

Figure 3: The simulated velocity distributions for signal samples with $|q|=2 e$ and mass 50,200 and $600 \mathrm{GeV}$.

In addition, the multiple electric charges of the particles influence the momentum reconstruction designed for $|q|=1 e$. Particles with higher charges bend more in the magnetic field leading to a misreconstruction of

$$
p_{\text {reco }}=\frac{p_{\text {true }}}{|q|},
$$

where $p_{\text {reco }}$ denotes the reconstructed momentum value, $p_{\text {true }}$ the true momentum of the particle and $|q|$ the absolute value of the charge. As a consequence, the selection efficiency is significantly reduced for signal samples with high charges.

\section{Results}

No signal candidates were observed in the signal regions in agreement with the SM expectation. The resulting 95\% CL upper cross section limits are shown in figure 4 alongside the predicted cross sections in the simplified DrellYan model used in the Monte Carlo production. The cross section limits lie in the range of $10^{-2}-10^{-1} \mathrm{pb}$, where the strongest cross section limit is observed for $|q|=2 e$.

From the crossings of the predicted and observed cross sections mass exclusion limits can be derived. The simplified Drell-Yan production model is excluded for particle masses in the range from $50 \mathrm{GeV}$ to $430,480,490,470$ and $420 \mathrm{GeV}$ for charge $|q|=$ $2,3,4,5$ and $6 e$, respectively, summarized in table 1 .

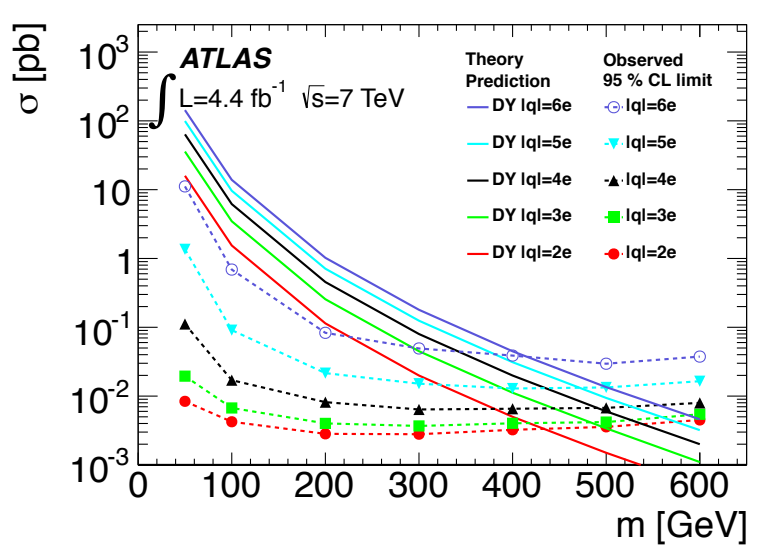

Figure 4: The observed 95\% CL upper cross section limits compared to the cross sections of the simplified Drell-Yan production model.

Table 1: Mass exclusion limits for the simplified Drell-Yan production model.

\begin{tabular}{llllll}
\hline$|q|[\mathrm{e}]$ & 2 & 3 & 4 & 5 & 6 \\
\hline$m_{\text {limit }}[\mathrm{GeV}]$ & 430 & 480 & 490 & 470 & 420 \\
\hline
\end{tabular}

\section{Summary}

A search for stable massive particles with electric charges of $|q|=2 e$ to $|q|=6 e$ is performed with the ATLAS detector at the LHC. A dataset of $4.4 \mathrm{fb}^{-1}$ is searched for highly ionization muon signatures. No deviation from the standard model expectations is observed and 95\% CL upper cross section limits on the order of $O(0.01-0.1 \mathrm{pb})$ are set. This result represents the first limit on the production cross section of stable massive particles with charges $|q|=2 e$ to $|q|=5 e$ published by the ATLAS collaboration and improves a previous limit on particles with $|q|=6 e$ by two orders of magnitude.

\section{References}

[1] ATLAS Collaboration, PLB 722, 305-323 (2013).

[2] ATLAS Collaboration, JINST 3 S08003 (2008).

[3] L. Evans and P. Bryant, JINST 3 S08001 (2008).

[4] ATLAS Collaboration, PLB 698, 353-370 (2011).

[5] C. A. Stephan, J. Phys. A 39 (2006) .

[6] R. Foadi, M. T. Frandsen, T. A. Ryttov, and F. Sannino, Phys. Rev. D 76 (2007). 